\title{
Recycling strange stars to millisecond periods
}

\author{
J. L. Zdunik ${ }^{1}$, P. Haensel ${ }^{1,2}$, and E. Gourgoulhon ${ }^{2}$ \\ 1 N. Copernicus Astronomical Center, Polish Academy of Sciences, Bartycka 18, 00-716 Warszawa, Poland \\ 2 Département d'Astrophysique Relativiste et de Cosmologie - UMR 8629 du CNRS, Observatoire de Paris, \\ 92195 Meudon Cedex, France \\ e-mail: haensel@camk.edu.pl; Eric.Gourgoulhon@obspm.fr
}

Received 28 August 2001 / Accepted 24 October 2001

\begin{abstract}
Recycling strange stars to millisecond periods is studied within the framework of general relativity. We employ equations of state of strange quark matter based on the MIT Bag Model, with massive strange quarks and lowest order QCD interactions. The presence of the crust of normal matter is taken into account, with a bottom density assumed to be equal to the neutron-drip one. The calculations are performed by solving the exact 2-D equations for rigidly rotating stationary configurations in general relativity. Evolutionary tracks of accreting strange stars are computed, and their dependence on the initial strange star mass and on the fraction of the angular momentum transferred to the star from the infalling matter, is studied. The differences between recycling strange stars and neutron stars are pointed out.
\end{abstract}

Key words. dense matter - equation of state - stars: neutron - stars: binaries: general

\section{Introduction}

The problem of the spin up of the neutron (or strange) stars is an important element of the studies of recycled pulsars. It is widely believed that millisecond pulsars $(P<10 \mathrm{~ms})$ are old neutron stars (age $\gtrsim 10^{9} \mathrm{y}$ ), with low magnetic field $\left(B \lesssim 10^{9} \mathrm{G}\right)$, which had been spun up by the accretion when they were members of the low-mass binaries (see, e.g., van den Heuvel \& Rappaport 1992). This evolutionary scenario of formation of millisecond pulsars recently obtained some observational support with the discovery of the first accretion-powered millisecond pulsar SAX J1808.4-3658 (Wijnands \& van der Klis 1998).

The detailed theoretical modeling of the spin-up scenario requires the solution of several specific problems that involve the knowledge of the neutron star structure, that in turn depends on the equation of state of neutron star matter. In particular, one has to determine the accreted mass needed to spin up a compact star to the observed millisecond periods, within a reasonable time, in low-mass $\mathrm{X}$-ray binaries. Finally, one would like to know what limits the maximum accreted mass - whether the upper bound results from the mass-shedding limit, or from some instability against gravitational collapse. The answers to all these questions depend on the (largely unknown) equation of state of dense matter at supranuclear density.

Send offprint requests to: J. L. Zdunik, e-mail: jlz@camk.edu.pl
Kluźniak \& Wagoner (1985) studied the spin up of an accreting neutron star (NS) for several equations of state of dense matter, using the lowest-order expansions in stellar angular momentum (slow rotation approximation, pioneered by Hartle 1967). Cook et al. (1994b, hereafter CST) modeled the recycling of NS without any restriction on neutron-star rotation, using their previous results for stationary rigidly rotating stellar configurations, which were obtained in the framework of general relativity for fourteen equations of state of dense matter (Cook et al. 1994c). A semianalytical model, based on the CST results, has been used by Burderi et al. (1999) to study the dependence of the recycling scenario on the magnetic field.

A hypothesis that the true ground state of matter is a deconfined self-bound quark plasma (Bodmer 1971; Witten 1984), called strange matter, implies the possibility of the existence of strange quark stars (Witten 1984; first detailed models in Haensel et al. 1986; Alcock et al. 1986). Recently, it has been argued that the accreting compact objects in some low-mass X-ray binaries could be strange stars (SS), and not NS (Bombaci 1997; Li et al. 1999a,b).

Previous studies of the properties of rotating strange stars pointed out significant, and in some cases strong and even qualitative differences between rapidly rotating SS and NS. In particular, the difference in the distribution of matter in the interior of the rapidly rotating NS and SS was shown to imply a significant difference in the outer spacetime. Specifically, qualitative differences in the properties of the innermost stable circular orbit (ISCO) around 
these two classes of rapidly rotating compact objects have been found (Stergioulas et al. 1999; Zdunik et al. 2000; Zdunik \& Gourgoulhon 2001). As shown in Zdunik et al. (2001, hereafter Paper I), a careful treatment of the crust of SS to some extent softens these conclusions, but important differences still remain. Recently, parameters of marginally stable orbits and properties of the accretion disk around SS have been studied, and compared with those around NS, by Bhattacharyya et al. (2001).

Differences between rapid rotation of SS and NS, as well as those between properties of the space-time around them, indicate the need for a study of differences between recycling by accretion of SS and NS. In the present paper we study spin-up of SS by accretion of matter from the innermost stable circular orbit. We neglect the effect of the magnetic field, accretion, and radiation drag on the location of the ISCO, and on the infall of accreting matter, which is justified for $B \lesssim 10^{9} \mathrm{G}$ and $\dot{M} \ll \dot{M}_{\mathrm{Edd}}$. Our approach is thus similar to that of CST. We have recently calculated the models of rotating strange stars with crust in general relativity (Paper I). Using the method described in Paper I, we determine the parameters necessary to calculate the motion of the matter accreting from the ISCO in the equatorial plane. The equation of state of strange matter, as well as the method for solving the relevant equations in general relativity, are briefly described in the first part of Sect. 2. The remaining part of Sect. 2 is devoted to the formulation of the basic equations governing the evolution of an accreting SS. Numerical results of computation of the evolutionary tracts of accreting SS are presented in Sect. 3, where we also study the basic differences between spin-up by accretion of SS and NS. Finally, Sect. 4 is devoted to the discussion of our results and gives some conclusions.

\section{Calculations}

The equation of state and calculational method for the stellar model are the same as that used in Paper I. Our EOS of strange matter, composed of massless $u$, d quarks, and massive s quarks, is based on the MIT Bag Model with the standard values of the Bag Model parameters for strange matter: bag constant $B=56 \mathrm{MeV} / \mathrm{fm}^{3}$, mass of the strange quark $m_{\mathrm{s}}=200 \mathrm{MeV} / c^{2}$, and QCD coupling constant $\alpha_{\mathrm{c}}=0.2$ (Farhi \& Jaffe 1984; Haensel et al. 1986; Alcock et al. 1986). This EOS of strange quark matter (called SQM1 in Paper I) had been also used in Zdunik et al. (2000) and Zdunik \& Gourgoulhon (2001). It yields an energy per unit baryon number at zero pressure $E_{0}=918.8 \mathrm{MeV}<E\left({ }^{56} \mathrm{Fe}\right)=930.4 \mathrm{MeV}$. The maximum allowable mass for static strange stars is $M_{\max }^{\text {stat }}=1.8 M_{\odot}$. The crust in our model is described by the BPS EOS below the neutron drip (Baym et al. 1970).

The general relativistic models of stationary rotating strange stars have been calculated by means of the code developed by Gourgoulhon et al. (1999), which relies on the multi-domain spectral method introduced in Bonazzola et al. (1998). In the present calculation we use
4 spatial domains (for details see Paper I). We supplemented the version of the code used in Paper I with the equations determining the specific angular momentum $l_{\mathrm{ms}}$ and specific energy $e_{\mathrm{ms}}$ of a test particle falling from the ISCO to the surface of the star (see the Appendix). We determine also the angle $\beta$, at which the particle falling freely from the ISCO hits the surface of the star,

$\tan (\beta)=\frac{p^{\widehat{r}}}{p^{\widehat{\varphi}}}$

where $p^{\hat{r}}, p^{\hat{\varphi}}$ are radial and tangential momentum of the infalling test particle, in the frame comoving with the matter at the stellar surface. For energetic considerations (heating of the surface), as well as for determining the probability of crossing the electrostatic barrier at the surface of SS, the crucial parameter is also the energy of the particle as seen by the observer comoving with the star. This energy is defined by the time component of the particle 4-momentum: $E=p^{\widehat{t}}$. The equations defining the parameters of the particle hitting the surface of the star are presented in the Appendix.

The evolutionary tracks of the accreting SS are calculated using the simplest scenario in which the increase of the angular momentum of the star is related to the accreted rest mass increment by

$\delta J=x_{l} l_{\mathrm{ms}} \delta M_{\mathrm{B}}$

where we introduced the parameter $x_{l}$ - the fraction of the total angular momentum of the particle transferred to the star. In the idealized, limiting case the accreted mass element transfers all its angular momentum to the star $\left(x_{l}=1\right)$. Equation (2) allows us to determine the total angular momentum $J$ and total rest (baryon) mass $M_{\mathrm{B}}$, after accreting matter of rest mass $\Delta M_{\mathrm{B}}$ :

$M_{\mathrm{B}}=M_{\mathrm{B}, 0}+\Delta M_{\mathrm{B}}$,

$J=J_{0}+\int_{0}^{\Delta M_{\mathrm{B}}} x_{l} l_{\mathrm{ms}}\left(M_{\mathrm{B}}, J\right) \mathrm{d} M_{\mathrm{B}}$,

where $M_{\mathrm{B} 0}$ and $J_{0}$ are initial baryon mass and angular momentum of the star, respectively. The values of $M_{\mathrm{B}}$ and $J$ determine then in a unique way the instantaneous configurations of accreting star, which form a one parameter family of rigidly rotating configurations, labeled by $M_{\mathrm{B}}$. These configurations form evolutionary tracks of accreting SS. The evolutionary tracks will be labeled by the gravitational mass $M_{0}$ of the initial configuration with baryon mass $M_{\mathrm{B}, 0}$. This initial configuration is assumed to be nonrotating, $J_{0}=0$.

For the star accreting at the rate $\dot{M}_{\mathrm{B}}$ (as seen by a distant observer), the time evolution of $J$ is given by the equation

$\frac{\mathrm{d} J}{\mathrm{~d} t}=x_{l} l_{\mathrm{ms}} \dot{M}_{\mathrm{B}}$

resulting directly from Eq. (2), where $t$ is the time measured at infinity. 


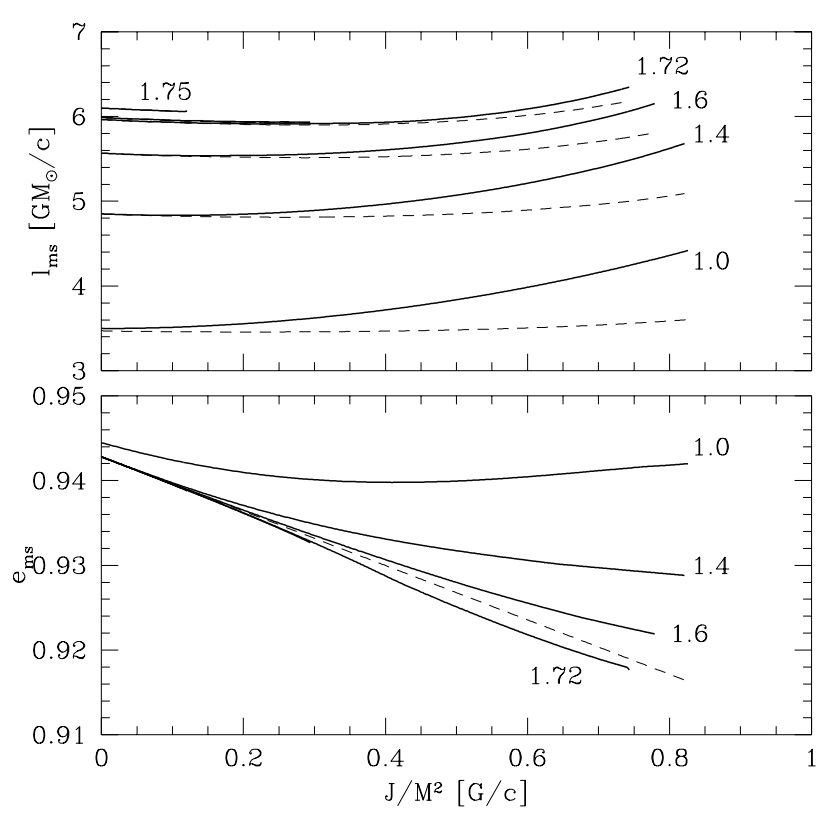

Fig. 1. Angular momentum and energy (per unit mass) of a particle at the marginally stable orbit along an evolutionary track for initial gravitational masses 1, 1.4,1.6, 1.72, 1.73, $1.75 M_{\odot}$ and linear approximation in $j=J c / G M^{2}$ of Kluźniak \& Wagoner (1985) (dashed). In the case of energy the curves for $1.73,1.75 M_{\odot}$ cannot be graphically distinguished from the upper part of the $1.72 M_{\odot}$ curve. The values of $l_{\mathrm{ms}}$ and $e_{\mathrm{ms}}$ for nonrotating configuration are $\sqrt{12} G M / c$ and $2 \sqrt{2} / 3$, respectively (except for the $1 M_{\odot}$ case, where the ISCO is defined by the stellar radius).

\section{Results}

\subsection{Changes of $I_{\mathrm{ms}}, e_{\mathrm{ms}}$ along the evolutionary tracks}

As one can see from Eq. (5), the quantity determining the evolution of an accreting star is the specific angular momentum of the particle at the ISCO: $l_{\mathrm{ms}}$. In the case of spherical, nonrotating configuration with $R<R_{\mathrm{ms}}$, the value obtained for the Schwarzschild metric is $\sqrt{12} \mathrm{GM} / \mathrm{c}$. For a rotating star, inclusion of the lowest order correction in a dimensionless parameter $j=J c / G M^{2}$ leads to the formula derived by Kluźniak \& Wagoner (1985): $\sqrt{12}\left(1-\frac{1}{3} \sqrt{\frac{2}{3}} j\right) G M / c$. Higher-order expansions in $j$ can be found in Shibata \& Sasaki (1998) (Eq. (B2) of their paper, where $j$ is denoted by $q$ ). In this article, we use instead the exact formula for $l_{\mathrm{ms}}$, as given in the Appendix.

In Fig. 1 we present the value of the angular momentum and energy of the test particle at the ISCO along evolutionary tracks. The approximate formula underestimates the value of $l_{\mathrm{ms}}$ and the actual spin up of the star is larger than predicted by the linear approximation (in j) of Kluźniak \& Wagoner (1985). The energy of the test particle at the ISCO, $e_{\mathrm{ms}}$, is smaller than that given by the linear approximation in $j$ for high masses only (the initial mass $\left.M_{0}>1.7 M_{\odot}\right)$. This is due to the fact that the next order terms in the expansion of $e_{\mathrm{ms}}$ and $l_{\mathrm{ms}}$ in $j$ are negative, while simultaneously rotational deformation of the star leads to the increase of both $e_{\mathrm{ms}}$ and $l_{\mathrm{ms}}$.

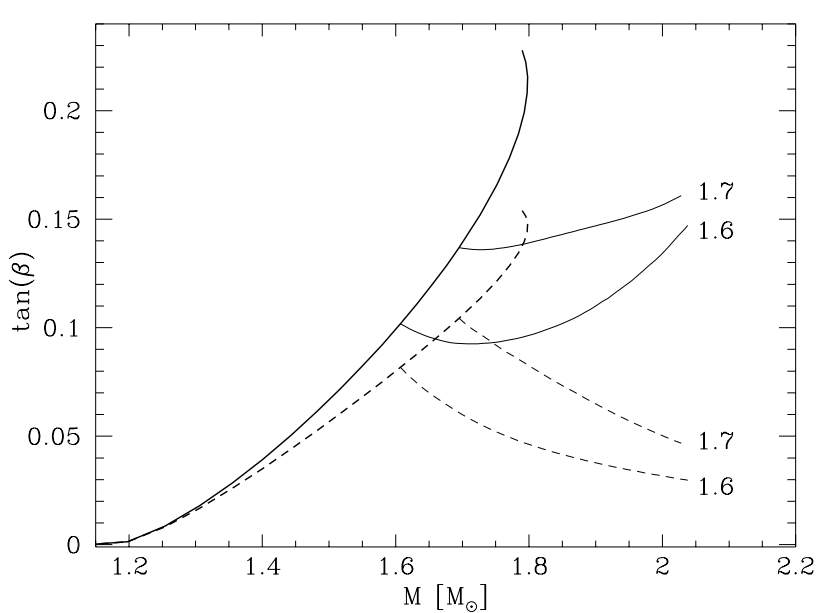

Fig. 2. The ratio $p^{\hat{r}} / p^{\hat{\varphi}}$, equal to the tangent of the incidence (impact) angle $\beta$, at which a test particle falling freely from the marginally stable orbit hits the stellar surface. Thick solid line - exact results for nonrotating configurations up to the maximum mass. Thin solid line - evolutionary tracks for $M_{0}=1.6 M_{\odot}$ and $M_{0}=1.7 M_{\odot}$. Dashed lines - same quantities, calculated using the approximate formula (Kluźniak \& Wagoner 1985). Evolutionary tracks terminate at the massshedding limit.

The negative correction, due to $j$, dominates only for the most massive stars, when the deformation due to the rotation is relatively small because of the very strong gravitational pull. Such a cancellation is not so pronounced for $e_{\mathrm{ms}}$, because there the relative magnitude of the $j^{2}$ correction is about twice that for $l_{\mathrm{ms}}$ (see Shibata \& Sasaki 1998, Eqs. (B1), (B2)).

Figure 2 shows the value of the tangent of the incidence (hitting) angle of the accreted matter, falling freely from the marginally stable orbit, as measured in the rest frame comoving with the stellar surface. The deviation of the approximate formula of Kluźniak \& Wagoner (1985) from the exact results is quite large, reaching $50 \%$ at maximum mass of the nonrotating configurations, and is even greater for rapidly rotating massive stars. The main reason for this discrepancy is the large gap between the stellar surface and marginally stable orbit for strange stars. For instance, at $M=M_{\max }$ and $\nu_{\text {rot }}=0$ we have $R \simeq 10 \mathrm{~km}$ and $R_{\mathrm{ms}} \simeq 16 \mathrm{~km}$, so that the linear approximation of the expansion of $\tan \beta$ in $\left(R_{\mathrm{ms}}-R\right) / R$, used by Kluźniak \& Wagoner (1985), is unjustified.

In Fig. 3 we present the value of the energy of the infalling particle (minus its rest mass $m_{0} c^{2}$ ) as seen by the observer at the stellar surface. This energy can be interpreted as the kinetic energy of the particle which should be lost as it gets a part of the star. As one can expect the energy tends to 0 as we are approaching Keplerian limit with $R>R_{\mathrm{ms}}$ (left part of $\nu_{\mathrm{rot}}=1000 \mathrm{~Hz}$ curve), since then the Keplerian velocity at the equator is equal to the velocity of the rotation. The exact results are compared with the approximate Newtonian formula (with a correction 


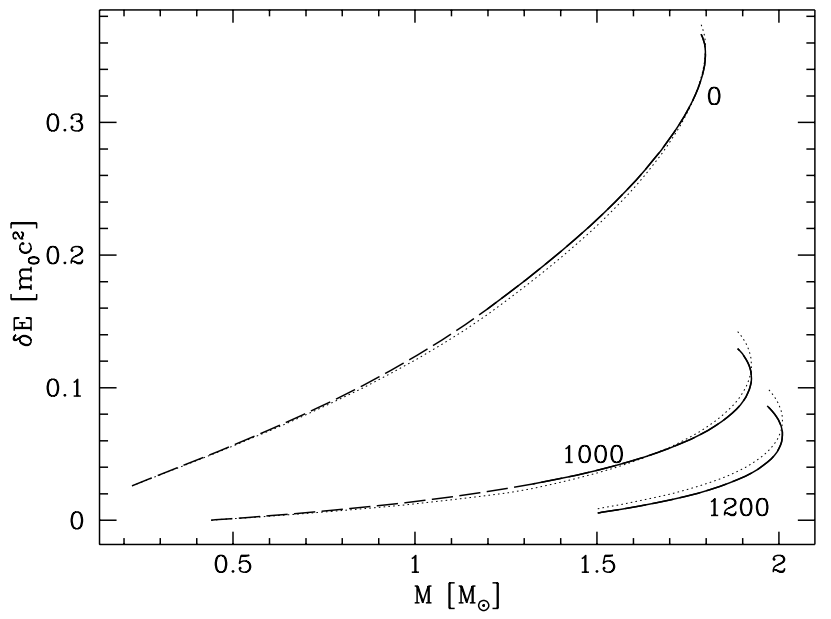

Fig. 3. Kinetic energy of a test particle falling freely from the marginally stable orbit as it hits the stellar surface (in the frame comoving with the surface of the star). The curves correspond to fixed rotational frequencies: $\nu_{\text {rot }}=0,1000,1200 \mathrm{~Hz}$. Thick lines - exact results (dashed part - ISCO defined by the stellar radius). Thin dotted lines - nonrelativistic approximation with relativistic time correction (see text).

involving the relativistic time delay which changes the velocities as seen by the observer at the massive star):

$\delta E=\frac{1}{2} \frac{\left(v_{\mathrm{ms}}-v\right)^{2}}{\left(1-2 G M / R c^{2}\right)}+\frac{G M}{R}\left(1-\frac{R}{R_{\mathrm{ms}}}\right)$,

where $v=\Omega R$ is the velocity of the star at the equator and $v_{\mathrm{ms}}=\Omega_{\mathrm{ms}} R_{\mathrm{ms}}$ is the velocity of the particle at the marginally stable orbit. This formula approximates $\delta E$ with a surprisingly small error.

\subsection{Accreted crust}

Standard models of strange matter predict the existence of an electron layer, which extends beyond the sharp quark surface by some $\sim 300-400 \mathrm{fm}$. The electrons in this layer are bound to the quark surface by a positive Coulomb potential, produced by quarks, of height $\sim 30-50 \mathrm{MeV}$. Spherical accretion of a plasma onto a SS with low magnetic field ( $B \lesssim 10^{9} \mathrm{G}$ ) was studied in (Haensel \& Zdunik 1991; Miralda-Escudé et al. 1990). In the case of spherically symmetric accretion, protons at the instant of hitting a bare (no crust) SS surface have an energy many times larger than the height of the Coulomb barrier, and traverse it with a large probability. After being absorbed by the strange matter, the protons (nucleons) dissolve into quarks in an exothermic process.

As we see in Fig. 2, in the case of accretion from the ISCO, nuclei in the infalling plasma hit the SS surface at a very oblique angle $\beta \lesssim 10^{\circ}$; they should therefore be efficiently deflected by the electrostatic barrier. The kinetic energy of a proton hitting the SS surface can be estimated as $0-300 \mathrm{MeV}$ (Fig. 3), and depends very sensitively on the value of $\Omega$. The largest value of $\delta E$ corresponds to the nonrotating star with maximum mass, since then the two components defining this energy are very large: the

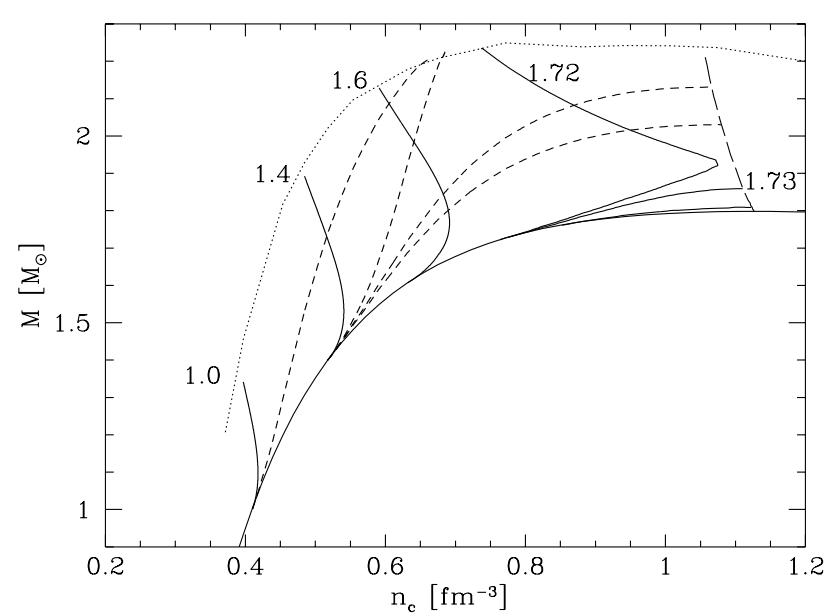

Fig. 4. Gravitational mass versus central baryon number density along several evolutionary tracks of accreting strange stars. Solid lines correspond to the typical case - all angular momentum of matter falling from the marginally stable orbit is transferred to the star $\left(x_{l}=1\right)$. Dashed lines represent three cases for a $M_{0}=1.4 M_{\odot} \operatorname{star}\left(x_{l}=0.66,0.55,0.5\right.$ from the left to the right), and the case $x_{l}=0.5$ for a $M_{0}=1 M_{\odot}$ star. Longdashed, nearly vertical line at maximum mass is defined by the onset of instability with respect to axisymmetric perturbations (see the text), dotted line - Keplerian (mass shedding) limit.

velocity of the freely falling particle relative to the nonrotating star (the kinetic energy) and the gap between the marginally stable orbit and stellar surface (the potential energy). However a very small incidence angle increases strongly the effective thickness of the Coulomb barrier to be penetrated, as well as the effective thickness of the electron layer to be traversed (implying therefore higher energy loss). All in all, infalling protons (or more generally - nuclei) are not able to penetrate the Coulomb barrier, and one expects a gradual building-up of a normal-matter envelope (crust) on the top of the strange matter surface. The bottom density of the accreted crust is limited by the neutron-drip. Further accretion induces irreversible absorption of neutrons by strange matter, and their fusion with strange matter in a strong-interaction process $\mathrm{n} \longrightarrow 2 \mathrm{~d}+\mathrm{u}$, followed by the weak-interaction process $\mathrm{u}+\mathrm{d} \longrightarrow \mathrm{s}+\mathrm{u}$. These reactions lead to an additional heat release (deconfinement heating) at the crust-strange matter interface, of some $30-40 \mathrm{MeV}$ per accreted nucleon.

\subsection{SS parameters along evolutionary tracks}

In Fig. 4 we plot the gravitational mass of the star as a function of the central density, for different choices of $M_{\mathrm{B}, 0}$ and for $J_{0}=0$. This figure can be directly compared with Fig. 1 of CST, obtained for NS. The main differences between neutron and strange stars spun up by the accretion are the following:

- the difference between maximum masses for rotating and nonrotating SS is more than $0.4 M_{\odot}$ compared with at most $0.3 M_{\odot}$ for NS; 


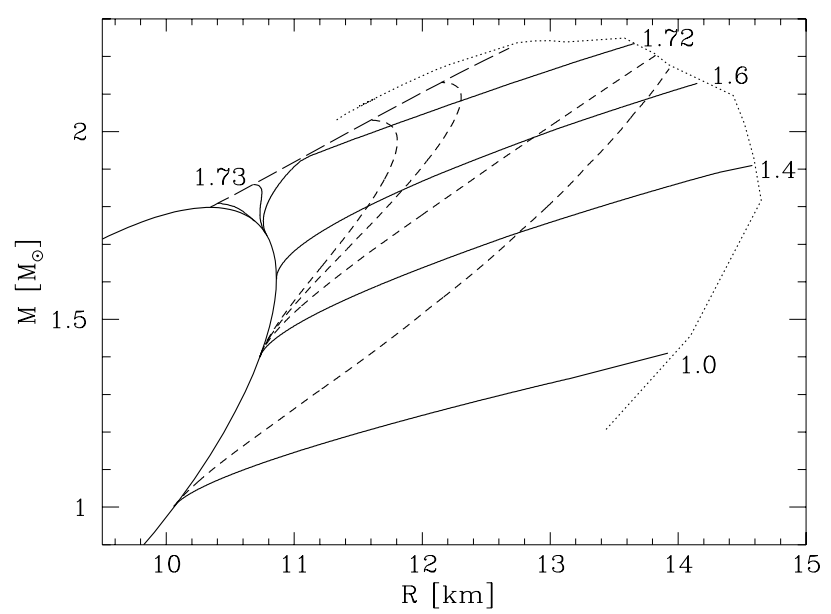

Fig. 5. Mass versus the (circumferential) equatorial radius, along several evolutionary tracks of accreting strange stars. Notations as in Fig. 4.

- the specific angular momentum $l_{\mathrm{ms}}$ for SS is sufficiently high to result in a characteristic "turning point" of the evolutionary track in the $\rho_{\mathrm{c}}-M$ plane, at which the central density of accreting SS starts to decrease. The effect of the increasing angular momentum (leading to the deformation of the star and decrease of $\rho_{\mathrm{c}}$ ) starts there to be dominating over the the effect of the mass increase tending to increase the central density;

- the rapid increase of $J$, compared to NS, determines the final fate of the accreting SS: nearly all evolutionary sequences terminate at the mass-shedding limit. Instability with respect to axisymmetric perturbations (which would eventually lead to the collapse of accreting SS into a black hole) can be reached only for initial (static) mass very close to the maximum one for $x_{l}=1$; for our EOS this is restricted to the narrow range $1.73 M_{\odot}<M_{0}<M_{\max }=1.8 M_{\odot}$;

- in the case of accreting SS, there exists a region of stable rotating configurations with high $M$ and high $\rho_{\mathrm{c}}$, which cannot be reached via accretion of matter onto an initially static SS (unless $x_{l}<1$ );

- contrary to the case of NS, for astrophysically interesting masses (say, $M>M_{\odot}$ ), we have, during accretion, $R_{\mathrm{eq}}<R_{\mathrm{ms}}$. This means, that along the evolutionary track the marginally stable orbit is always located above the stellar surface. This conclusion is different from that presented in Paper I, because it refers here to the supramassive configurations, which have not been considered in our previous paper.

The mass-radius relations for accreting SS along evolutionary tracks are shown in Fig. 5. The region that cannot be reached by the accretion is not so spectacular as in the case of $n_{\mathrm{c}}-M$ dependence. It is clearly visible that stars which, due to the accretion, directly enter the region of instability with respect to axisymmetric perturbations (implying collapse to black hole) lie in a very narrow region $\left(1.9>M / M_{\odot}>1.73,11 \mathrm{~km}>R>10.2 \mathrm{~km}\right)$. However it should be noted that in principle it is possible

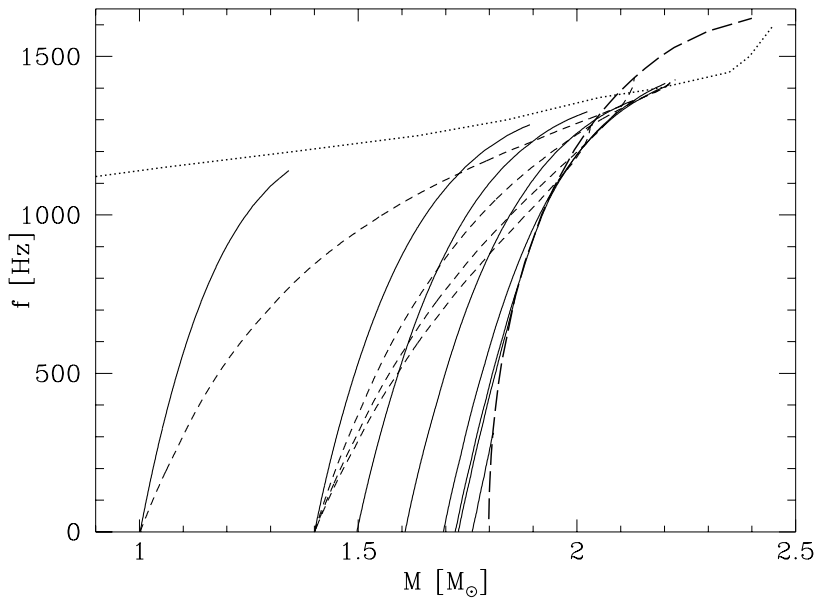

Fig. 6. Frequency of the rotation versus gravitational mass along several evolutionary tracks of accreting strange stars. Notations as in Fig. 4.

that stars spun up by the accretion to the rotational frequencies very close to the Keplerian one eventually are slowing down due to some processes leading to the angular momentum loss (violating of course Eq. (2)) and reach the region forbidden from the point of view of accretion.

In Fig. 6 we present rotational frequency and gravitational mass of the star - two quantities which could in principle be observed. It should be stressed that the point in the $f-M$ plane do not uniquely defines the rotating configuration. In particular the crossing point between the Keplerian (dotted) and the instability limit (thick dashed) curves at about $2.1 M_{\odot}, 1400 \mathrm{~Hz}$ do not correspond to the same star (the Keplerian limit star has a total angular momentum $J=4.2 G M_{\odot}^{2} / c$ and central density $n_{\mathrm{c}} \simeq 0.5 \mathrm{fm}^{-3}$ while the marginally stable star has $J=2.6 G M_{\odot}^{2} / c$ and $\left.n_{\mathrm{c}}=1.1 \mathrm{fm}^{-3}\right)$. As a consequence the region below the instability line and above the Keplerian limit (dotted) curve for supramassive stars cannot be treated as forbidden (stable rotating configurations exist there) although it can be reached by the accretion only in the case of $x_{l}<1$.

The existence of the region which cannot be reached by the accretion can be suitably discussed in the $J-M_{\mathrm{B}}$ plane - two basic parameters from the point of view of accretion (Fig. 7). The evolutionary tracks are defined by the requirement that the change of the stellar angular momentum is connected with the accreted mass via Eq. (2), i.e., the derivative $\mathrm{d} J / \mathrm{d} M_{\mathrm{B}}$ along evolutionary tracks is equal to $x_{l} l_{\mathrm{ms}}$. The allowed region is bounded by the Keplerian limit (high $J$ ) and by the instability with respect to axisymmetric perturbations (high $M_{\mathrm{B}}$ ) leading to the collapse. As it can be seen from Fig. 7 the accreting star eventually collapses if the evolutionary track crosses the instability limit (long dashed curve in Fig. 7), which is possible if $\mathrm{d} J / \mathrm{d} M_{\mathrm{B}}$ along this limit is larger than $\left(\mathrm{d} J / \mathrm{d} M_{\mathrm{B}}\right)_{\text {evol }}$ along the evolutionary track. For $x_{l}=1$ this condition limits the possible parameters of collapsing accreting SS to a very narrow range (high $M_{\mathrm{B}}$ and low $J$ ) 


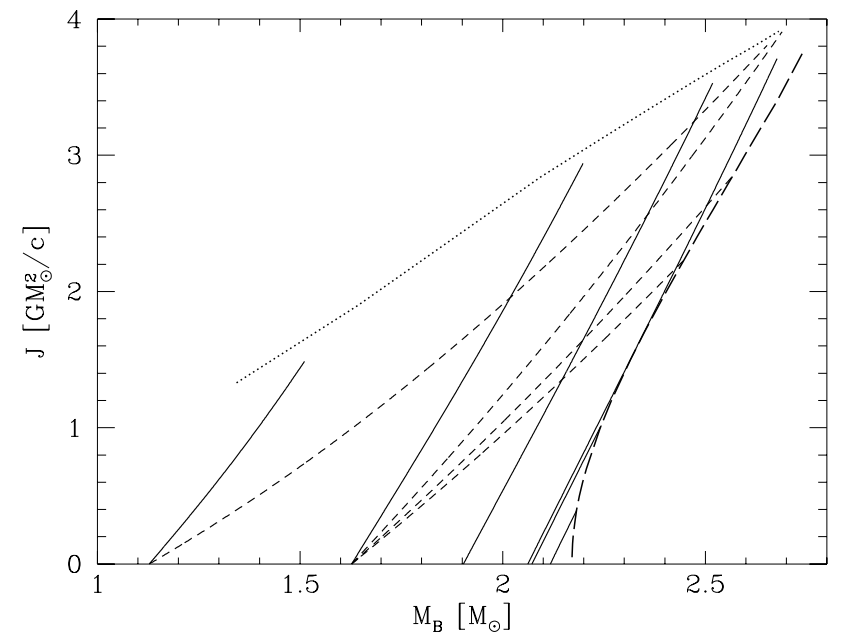

Fig. 7. Angular momentum of the star versus its baryon mass, along several evolutionary tracks of accreting strange stars. At each point, the slope (first derivative) of evolutionary curves is equal to the value of $x_{l} l_{\mathrm{ms}}$. Notations as in Fig. 4.

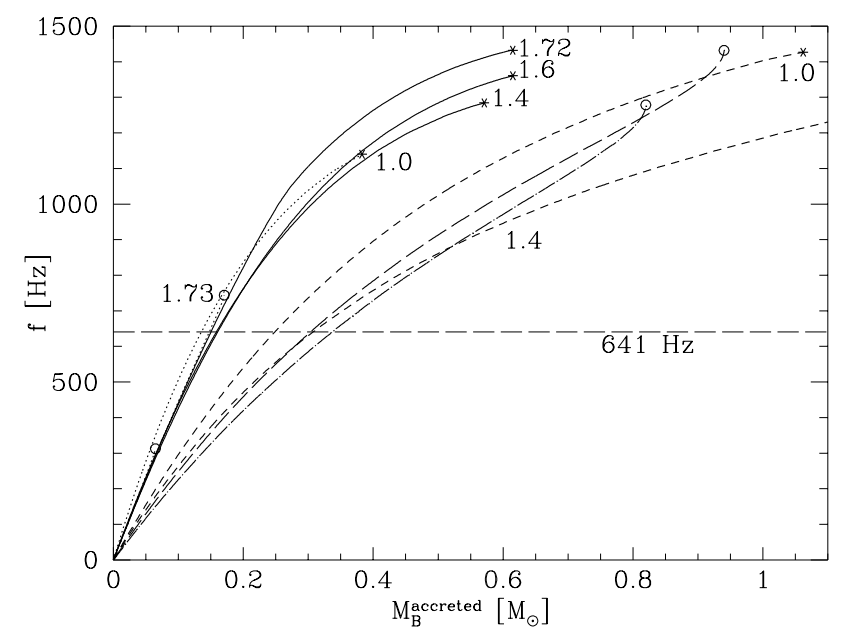

Fig. 8. Rotational frequency of rotating strange star as a function of the accreted baryon mass. Dotted lines $-M_{0}=$ $1,1.73,1.75 M_{\odot}$. Dashed lines correspond to $x_{l}=0.5$ for $M_{0}=1 M_{\odot}$ and $x_{l}=0.5$ (dashed), $x_{l}=0.55$ (dot-dashed), $x_{l}=0.66$ (long dash) for $M_{0}=1.4 M_{\odot}$. The last points of the each evolutionary track are labelled by the initial gravitational mass and denoted by a star (mass-shed limit) or circles (collapse).

whereas for a smaller $x_{l}$ all configurations on the instability curve can be reached by accretion. It should be noted that, contrary to Figs. 4 and 5, Fig. 7 presents stellar configurations in a non-unique way, i.e., above the longdashed curve there exist stable (lower $n_{\mathrm{c}}$, higher $R$ ) as well as unstable (higher $n_{\mathrm{c}}$, lower $R$ ) configurations. Stars below this curve do not exist, which is the direct consequence of this stability criterion (minimum $J$ at fixed $M_{\mathrm{B}}$ ).

Figure 8 shows the rotational frequency of a spunup SS versus the accreted baryon mass. The evolution of the rotational period depends critically on the fraction of the angular momentum at the ISCO, transferred to the star, $x_{l}$. On the contrary, the character of the spin-up

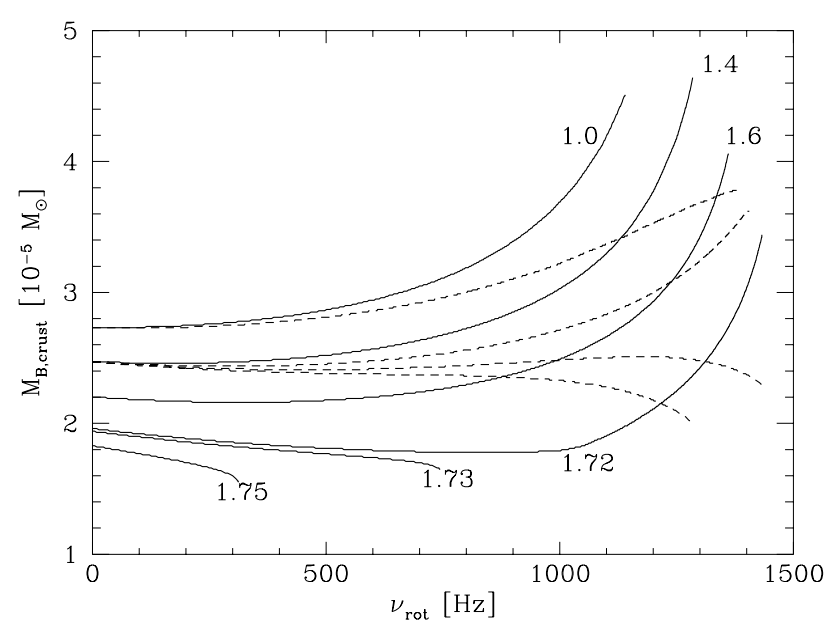

Fig. 9. Baryon mass of the crust along evolutionary tracks. $M_{0}=1,1.4,1.6,1.72,1.73,1.75 M_{\odot}$. Higher initial mass corresponds to lower $M_{\mathrm{B}, \mathrm{crust}}$. Dashed curves correspond to $x_{l}=0.5$ for $M_{0}=1 M_{\odot}$ and $x_{l}=0.5,0.55,0.66$ (from bottom to the top) for $M_{0}=1.4$.

depends rather weakly on the initial mass of the star. For $x_{l}=1$, the submillisecond periods are reached after accretion of about $0.3 M_{\odot}$. Accretion of $0.2 M_{\odot}$ is needed to spin up the SS to $1.56 \mathrm{~ms}(f=641 \mathrm{~Hz})$, the minimum observed period of a millisecond pulsar. Notice, that at a fixed $\dot{M}_{\mathrm{B}}$, the abscissa in Fig. 8 is proportional to the accretion time, $t_{\mathrm{acc}}=M_{\mathrm{B}, \text { acc }} / \dot{M}_{\mathrm{B}}$.

An interesting quantity, in the astrophysical context, is the accreted rest mass required to spin up the SS to the termination point of the evolutionary track (i.e., to the mass-shedding limit, or to the threshold for instability with respect to the axisymmetric perturbations, which results in collapse into a black hole). For the evolutionary tracks which eventually lead to collapse into a black hole, this maximum accreted mass depends sensitively on the initial mass $M_{0}$, i.e., how close $M_{0}$ is to the maximum allowable mass. For example, for $M_{0}=1.75 M_{\odot}$, the maximum accreted mass is only $M_{\mathrm{B} \text {,acc }}=0.07 M_{\odot}$, while for the lowest $M_{0}$, for which the evolutionary track terminates by instability with respect to axially symmetric perturbations, $M_{0}=1.723 M_{\odot}$, the SS accretes $M_{\mathrm{B}, \text { acc }}=0.2 M_{\odot}$ before collapsing into a black hole. The maximum accreted mass, required to spin up a SS from $\nu_{\text {rot }}=0$ to the massshedding limit $\nu_{\text {rot }}=\nu_{\mathrm{K}}$, practically does not depend on the initial mass (provided it is not too small, $M_{0}>1 M_{\odot}$ ), and is roughly equal $M_{\mathrm{B} \text {, acc }}=0.6 M_{\odot}$, i.e., is larger by some $50 \%$ than in the case of neutron stars (see CST).

The parameters of the crust (the baryon mass and the thickness) are presented in Figs. 9, 10. The interesting difference with respect to the curves presented in Paper I for a fixed baryon mass of the star $M_{\mathrm{B}}$ is the slower increase (or even decrease) of $M_{\mathrm{B}, \text { crust }}$ and $\Delta R_{\text {crust }}$ with increasing $\nu_{\text {rot }}$. This is caused by the increase of the SS mass during accretion: a larger mass corresponds to higher gravitational pull and smaller crust (Paper I). Although the rotation results in the increase of $M_{\mathrm{B}, \text { crust }}$ and $\Delta R_{\text {crust }}$ at 


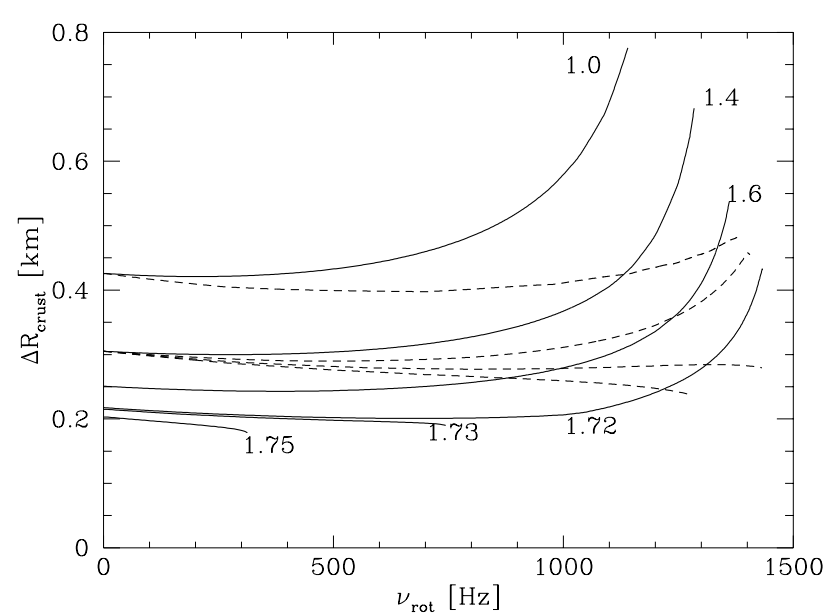

Fig. 10. Thickness of the crust along evolutionary tracks. Notations as in Fig. 9.

slow rotation rates the mass increase can dominate, leading to the shrinking of the crust. Of course, the effect of the mass increase is more important, if the accreted mass is connected with a slower acceleration of the rotation (i.e. $\left.x_{l}<1\right)$. For example, for $x_{l}=0.5$ the size of the crust of an accreting $M_{0}=1.4 M_{\odot}$ SS star is continuously decreasing along the evolutionary track.

\section{Discussion and conclusion}

Our study of SS recycling by accretion have shown significant differences with respect to the case of NS. These differences stem both from the basic difference in the internal structure (density profile) and from the difference in the outer space-time around rotating SS and NS. The latter implies differences in the properties of the marginally stable orbit, from which the matter is falling onto the compact object, transferring its angular momentum to the star. Our calculations have been performed for a specific EOS of strange matter. However, the qualitative features of evolutionary tracks, described in Sect. 3, are generic for SS, and do not depend on the specific EOS of self-bound strange quark matter.

The standard models of strange matter (a recent review can be found in Madsen 1999), predict the presence of a small fraction of electrons, which is due to the $\mathrm{SU}(3)$ symmetry breaking in the quark sector $\left(m_{\mathrm{s}} \gg m_{\mathrm{u}}, m_{\mathrm{d}}\right)$. The electrostatic potential, which bounds electrons to SS, produces then a huge outward-directed electric field, which is necessary to support a crust of normal matter on the strange matter core. Using such a strange matter model, one is able to study the formation and evolution of the crust during the accretion process.

Very recently, Rajagopal \& Wilczek (2001) demonstrated that quark matter in the color-flavor locked (CFL) superconducting phase of QCD is strictly neutral, despite unequal quark masses: $n_{\mathrm{u}}=n_{\mathrm{d}}=n_{\mathrm{s}}$. If strange matter is actually in the CFL superconducting phase, then SS would be bare, with no electrons, and with a surface thickness of $\sim \hbar c / \mu$, where $\mu \sim 400 \mathrm{MeV}$ is the quark chemical potential. In absence of any repulsive Coulomb barrier, the stable coexistence of normal and quark matter would then be excluded. All accreted matter would be then absorbed in an exothermic process. However, the effect on the evolutionary tracks of accreting SS would be small. The gap $R_{\mathrm{ms}}-R_{\text {eq }}$ would be larger, and the shedding-limit terminations would shift to somewhat higher frequencies, allowing for accreting of a larger amount of matter. However, the parameters of the marginally stable orbit, and the rate of spin-up of accreting SS would not change, because the maximum fraction of stellar mass and moment of inertia contained in a normal crust is at most $10^{-4}$.

Acknowledgements. This research was partially supported by the KBN grant No. 5P03D.020.20 and by the CNRS/PAN program Jumelage Astrophysique.

\section{Appendix A: Free fall from marginally stable orbit - impact parameters}

In our considerations the metric can be written in the form:

$$
\begin{aligned}
\mathrm{d} s^{2}= & -N^{2} \mathrm{~d} t^{2}+B^{2} r^{2} \sin ^{2} \theta\left(\mathrm{d} \varphi-N^{\varphi} \mathrm{d} t\right)^{2} \\
& +A^{2}\left(\mathrm{~d} r^{2}+r^{2} \mathrm{~d} \varphi^{2}\right)
\end{aligned}
$$

where $N, N^{\varphi}, A$ and $B$ are four metric functions of $(r, \theta)$. For a complete description of the coordinate system, metric functions and relativistic equations see Bonazzola et al. (1993) and Gourgoulhon et al. (1999). Here we present only the equations for the parameters of the particle falling freely from the marginally stable orbit (ISCO).

The location (coordinate $r_{\mathrm{ms}}$ ) and orbital frequency $\Omega_{\mathrm{ms}}$ of the ISCO are obtained by solving the equations presented in e.g. Cook et al. (1994a), Datta et al. (1998), Schaab \& Weigel (1999). For any particle falling along a geodesic from the ISCO to the stellar surface, the conserved quantities are the specific energy

$e_{\mathrm{ms}}=-p_{t}=\Gamma_{\mathrm{ms}}\left(N+B r_{\mathrm{ms}} N^{\varphi} V_{\mathrm{ms}}\right)$

and the specific angular momentum

$l_{\mathrm{ms}}=p_{\varphi}=\Gamma_{\mathrm{ms}} B r_{\mathrm{ms}} V_{\mathrm{ms}}$,

where $p_{\mu}$ denotes the covariant components with respect to the coordinates $(t, r, \theta, \varphi)$ of the particle 4-momentum (per unit mass), $V_{\mathrm{ms}}$ is the particle 3 -velocity as measured by the zero-angular-momentum observer (ZAMO) and $\Gamma_{\mathrm{ms}}$ the corresponding Lorentz factor:

$V_{\mathrm{ms}}:=\frac{B}{N} r_{\mathrm{ms}}\left(\Omega_{\mathrm{ms}}-N^{\varphi}\right)$,

$\Gamma_{\mathrm{ms}}:=\left(1-V_{\mathrm{ms}}^{2}\right)^{-1 / 2}$.

In the above equation, all the metric factors are to be evaluated at the location of the ISCO.

Let us now compute the impact angle and energy of the particle as it reaches the stellar surface. First we construct an orthonormal tetrad connected with the observer 
comoving with surface of the star, the angular velocity of which is $\Omega$ (as seen from infinity). Let $\left(\boldsymbol{e}_{\widehat{\boldsymbol{t}}}, \boldsymbol{e}_{\widehat{\varphi}}, \boldsymbol{e}_{\widehat{\boldsymbol{r}}}, \boldsymbol{e}_{\widehat{\theta}}\right)$ denote this tetrad. It is expressed in terms of the the coordinate basis $\left(\boldsymbol{e}_{\boldsymbol{t}}, \boldsymbol{e}_{\boldsymbol{\varphi}}, \boldsymbol{e}_{\boldsymbol{r}}, \boldsymbol{e}_{\theta}\right)$ as follows:

$$
\begin{aligned}
& \boldsymbol{e}_{\widehat{\boldsymbol{t}}}=\boldsymbol{u}=\frac{\Gamma}{N} \boldsymbol{e}_{\boldsymbol{t}}+\Omega \frac{\Gamma}{N} \boldsymbol{e}_{\varphi} \\
& \boldsymbol{e}_{\widehat{\varphi}}=\frac{\Gamma}{N} U_{\mathrm{s}} \boldsymbol{e}_{\boldsymbol{t}}+\frac{\Gamma}{B r}\left(1+\frac{B r}{N} N^{\varphi} U_{\mathrm{s}}\right) \boldsymbol{e}_{\varphi} \\
& \boldsymbol{e}_{\widehat{\boldsymbol{r}}}=\frac{1}{A} \boldsymbol{e}_{\boldsymbol{r}} \\
& \boldsymbol{e}_{\widehat{\theta}}=\frac{1}{A r} \boldsymbol{e}_{\theta}
\end{aligned}
$$

where $\boldsymbol{u}$ is the 4 -velocity of the stellar matter, $U_{\mathrm{s}}$ its 3 -velocity with respect to the ZAMO,

$$
U_{\mathrm{s}}:=\frac{B r}{N}\left(\Omega-N^{\varphi}\right),
$$

and $\Gamma$ the corresponding Lorentz factor:

$$
\Gamma:=\left(1-U_{\mathrm{s}}^{2}\right)^{-1 / 2} \text {. }
$$

It is easy to check that this tetrad is orthonormal, i.e. $\boldsymbol{e}_{\widehat{\mu}} \cdot \boldsymbol{e}_{\widehat{\nu}}=\eta_{\mu \nu}=\operatorname{Diag}(-1,1,1,1)$.

The freely falling particle that hits the surface of the star has a 4-momentum $\boldsymbol{p}$ with two conserved components: the quantities $p_{t}=-e_{\mathrm{ms}}$ and $p_{\varphi}=l_{\mathrm{ms}}$ given by Eqs. (A.2) and (A.3). For a motion within the equatorial plane, the component $p_{\theta}$ vanishes. The component $p_{r}$ is obtained from the normalization relation $\boldsymbol{p} \cdot \boldsymbol{p}=-1$ :

$p_{r}^{2}=A^{2}\left[\frac{\left(e_{\mathrm{ms}}-N^{\varphi} l_{\mathrm{ms}}\right)^{2}}{N^{2}}-\frac{l_{\mathrm{ms}}^{2}}{B^{2} r^{2}}-1\right]$,

which of course is nonzero at the surface of the star unless the ISCO is determined by the stellar radius.

In the frame comoving with matter (tetrad A.6) the momentum of the particle is given by:

$$
\begin{aligned}
p^{\widehat{t}} & =-\boldsymbol{p} \cdot \boldsymbol{e}_{\widehat{\boldsymbol{t}}}=-\left(p_{\varphi}\left(\boldsymbol{e}_{\widehat{\boldsymbol{t}}}\right)^{\varphi}+p_{t}\left(\boldsymbol{e}_{\widehat{\boldsymbol{t}}}\right)^{t}\right)=\frac{\Gamma}{N}\left[e_{\mathrm{ms}}-\Omega l_{\mathrm{ms}}\right] \\
p^{\widehat{r}} & =\boldsymbol{p} \cdot \boldsymbol{e}_{\widehat{\boldsymbol{r}}}=p_{r}\left(\boldsymbol{e}_{\widehat{\boldsymbol{r}}}\right)^{r}=p_{r} / A \\
& =\sqrt{\frac{\left(e_{\mathrm{ms}}-N^{\varphi} l_{\mathrm{ms}}\right)^{2}}{N^{2}}-\frac{l_{\mathrm{ms}}^{2}}{B^{2} r^{2}}-1} \\
p^{\widehat{\varphi}} & =\boldsymbol{p} \cdot \boldsymbol{e}_{\widehat{\varphi}}=p_{\varphi}\left(\boldsymbol{e}_{\widehat{\varphi}}\right)^{\varphi}+p_{t}\left(\boldsymbol{e}_{\widehat{\varphi}}\right)^{t} \\
& =\frac{\Gamma l_{\mathrm{ms}}}{B r}\left[1+\frac{B r U_{\mathrm{s}}}{N}\left(N^{\varphi}-\frac{e_{\mathrm{ms}}}{l_{\mathrm{ms}}}\right)\right]
\end{aligned}
$$

The angle $\beta$ at which the particle falling freely from the marginally stable orbit hits the surface of the star is given by

$$
\tan \beta=\frac{p^{\widehat{r}}}{p^{\widehat{\varphi}}} .
$$

The energy release per unit mass due of infalling matter in the comoving frame is $p^{\widehat{t}}-1$, i.e.

$\delta E=\frac{\Gamma}{N}\left[e_{\mathrm{ms}}-\Omega l_{\mathrm{ms}}-N / \Gamma\right]$.
This is the value which should be used for the energy balance considerations at the stellar surface (heating of the matter). If we assume that all of this energy is emitted, this results in a luminosity (per unit accreted mass) seen by a distant observer $\delta E / u^{t}=e_{\mathrm{ms}}-\Omega l_{\mathrm{ms}}-N / \Gamma$, as it has recently been demonstrated by Sibgatulin \& Sunyaev (2000).

\section{References}

Alcock, C., Farhi, C. E., \& Olinto, A. 1986, ApJ, 310, 261

Baym, G., Pethick, C., \& Sutherland, P. 1971, ApJ, 170, 299

Bhattacharyya, S., Thampan, A. V., \& Bombaci, I. 2001, A\&A, 372,925

Bodmer, A. R. 1971, Phys. Rev. 4, 1601

Bombaci, I. 1997, Phys. Rev. C, 55, 1587

Bonazzola, S., Gourgoulhon, E., Salgado, M., \& Marck, J. A. 1993, A\&A, 278, 421

Bonazzola, S., Gourgoulhon, E., \& Marck, J. A. 1998, Phys. Rev. D, 58, 104020

Burderi, L., Possenti, A., Colpi, M., Di Salvo, T., \& D’Amico, N. 1999, ApJ, 519, 285

Cook, G. B., Shapiro, S. L., \& Teukolsky, S. A. 1994a, ApJ, 422,227

Cook, G. B., Shapiro, S. L., \& Teukolsky, S. A. 1994b, ApJ, 423, L117 (CST)

Cook, G. B., Shapiro, S. L., \& Teukolsky, S. A. 1994c, ApJ, 424,823

Datta, D., Thampan, A. V., \& Bombaci, I. 1998, A\&A, 334, 943

Farhi, E., \& Jaffe, R. L. 1984, Phys. Rev. D, 30, 2379

Gourgoulhon, E., Haensel, P., Livine, R., et al. 1999, A\&A, 349,851

Haensel, P., Zdunik, J. L., \& Schaeffer, R. 1986, A\&A, 160, 121

Haensel, P., \& Zdunik, J. L. 1991, Nucl. Phys. B (Proc. Suppl.), 24B, 139

Hartle, J. B. 1967, ApJ, 150, 1005

Kluźniak, W., \& Wagoner, R. V. 1985, ApJ, 297, 548

Li, X.-D., Bombaci, I., Dey, M., Dey, J., \& van den Heuvel, E. P. J. 1999a, Phys. Rev. Lett. 83, 3776

Li, X.-D., Ray, S., Dey, J., Dey, M., \& Bombaci, I. 1999b, ApJ, 527, L51

Madsen, J. 1999, in Hadrons in Dense Matter and Hadrosynthesis, Lect. Notes in Phys., ed. J. Cleymans (Springer-Verlag), 162

Miralda-Escudé, J., Haensel, P., \& Paczyński, B. 1990, ApJ, 362,572

Schaab, C., \& Weigel, M. K. 1999, MNRAS, 308, 718

Rajopagal, K., \& Wilczek, F. 2001, Phys. Rev. Lett., 86, 3492

Shibata, M., \& Sasaki, M. 1998, Phys. Rev. D, 58, 104011

Sibgatulin, N., \& Sunyaev, R. 2000, Astron. Lett., 26, 772

van den Heuvel, E. P. S., \& Rappaport, S. A. 1992, X-Ray binaries and the formation of Binary and Millisecond Pulsars (Dordrecht, Kluver)

Wijnands, R., \& van der Klis, M. 1998, Nature, 394, 344

Witten, E. 1984, Phys. Rev. D, 30, 272

Zdunik, J. L., \& Gourgoulhon, E. 2001, Phys. Rev. D, 63, 087501

Zdunik, J. L., Haensel, P., \& Gourgoulhon, E. 2001, A\&A, 372, 535, Paper I

Zdunik, J. L., Haensel, P., Gondek-Rosińska, D., \& Gourgoulhon, E. 2000, A\&A, 356, 612 